\title{
Polypharmacy among anabolic-androgenic steroid users: a descriptive metasynthesis
}

\author{
Dominic Sagoe ${ }^{1 *}$, Jim McVeigh ${ }^{2}$, Astrid Bjørnebekk ${ }^{3}$, Marie-Stella Essilfie" ${ }^{4}$ Cecilie Schou Andreassen ${ }^{1,5}$ \\ and Ståle Pallesen ${ }^{1}$
}

\begin{abstract}
Background: As far as we are aware, no previous systematic review and synthesis of the qualitative/descriptive literature on polypharmacy in anabolic-androgenic steroid(s) (AAS) users has been published.

Method: We systematically reviewed and synthesized qualitative/descriptive literature gathered from searches in electronic databases and by inspecting reference lists of relevant literature to investigate AAS users' polypharmacy. We adhered to the recommendations of the UK Economic and Social Research Council's qualitative research synthesis manual and the PRISMA guidelines.

Results: A total of 50 studies published between 1985 and 2014 were included in the analysis. Studies originated from 10 countries although most originated from United States $(n=22)$, followed by Sweden $(n=7)$, England only $(n=5)$, and the United Kingdom $(n=4)$. It was evident that prior to their debut, AAS users often used other licit and illicit substances. The main ancillary/supplementary substances used were alcohol, and cannabis/cannabinoids followed by cocaine, growth hormone, and human chorionic gonadotropin (hCG), amphetamine/meth, clenbuterol, ephedra/ephedrine, insulin, and thyroxine. Other popular substance classes were analgesics/opioids, dietary/nutritional supplements, and diuretics. Our classification of the various substances used by AAS users resulted in 13 main groups. These non-AAS substances were used mainly to enhance the effects of AAS, combat the side effects of AAS, and for recreational or relaxation purposes, as well as sexual enhancement.
\end{abstract}

Conclusions: Our findings corroborate previous suggestions of associations between AAS use and the use of other licit and illicit substances. Efforts must be intensified to combat the debilitating effects of AAS-associated polypharmacy.

Keywords: Anabolic-androgenic steroids, Ergogenic aids, Doping, Human enhancement drugs, IPEDs, Polypharmacy, Stacking, Metasynthesis, Qualitative research

\section{Introduction}

Anabolic-androgenic steroid(s) (AAS) refer to testosterone and its synthetic derivatives mainly used nonmedically for enhancing muscle growth and strength, boosting physical activity or sports performance, and for aesthetic purposes as well as for enhancing psychological well-being [1]. AAS are typically used in phases referred to as 'cycles': 'on cycles' referring to specific periods when the users administer AAS and 'off cycles' referring to an AAS-free phase intended to prevent tolerance towards AAS, lessen the possibility of side

\footnotetext{
* Correspondence: dominic.sagoe@psysp.uib.no

${ }^{1}$ Department of Psychosocial Science, University of Bergen, Christiesgate 12, 5015 Bergen, Norway

Full list of author information is available at the end of the article
}

effects, and allow recovery of natural hormonal functioning. During 'on cycles' users sometimes combine different injectable and oral AAS. This phenomenon is referred to as 'steroid stacking' or simply 'stacking' [2].

There is also a trend referred to as 'blast and cruise' or 'bridging' - a continuous 'on cycle' whereby many users never go off AAS but alternate between periods of high dose intake during a 'blast' phase, and low dose intake during a 'cruise' phase. Another way of administering AAS is called 'blitz-cycles', which implies rapidly changing AAS with the aim of preventing tolerance and androgen receptor down-regulation. Moreover, many users complement AAS use or stacking with the use of other substances. In this respect, AAS use has been found to be associated with the 
use of both licit and illicit substances in systematic reviews of predominantly quantitative literature [3,4].

It has been noted that one of the major drawbacks to successful AAS interventions is public health officials' failure to recognize AAS users' extensive pharmacological regimen [2]. A synthesis of the qualitative or descriptive literature on polypharmacy by AAS users is, both from a clinical and research perspective, important in order to increase the understanding of the polypharmacy often associated with AAS use. Such a literature review and synthesis is also valuable in terms of the development and strengthening of AAS use and harm reduction interventions as such investigation will deepen existing knowledge on the various substances used and the specific function they serve, which in some cases deviates significantly from their formal medical indications. Furthermore, results of such investigation would complement evidence emanating from a systematic review of mostly quantitative evidence [3] in the effort to elucidate the phenomenon of polysubstance use by AAS users. However, as far as we are aware, a systematic review and synthesis of the qualitative or descriptive literature on polypharmacy by AAS users has not been published.

Against this backdrop, we conducted the first systematic review and synthesis of the qualitative or descriptive studies presenting data on the use of other licit and illicit substances among AAS users. The research questions guiding the present study were: (a) what substances do AAS users report consuming prior to their AAS debut? (b) what ancillary or supplementary substances do AAS users report using? and (c) what reasons do AAS users assign for using these substances?

\section{Method}

\section{Search strategy and inclusion criteria}

We searched in PsycINFO, PubMed, ISI Web of Science, and Google Scholar for literature. For searches in PubMed and ISI Web of Science, 'anabolic steroid', 'doping', and 'performance enhancing drug', were each combined with 'interview', 'focus group', and 'qualitative'. These combinations were not practical in PsycINFO and Google Scholar as they produced voluminous redundant hits. Thus, 'anabolic steroid + doping + performance enhancing drug + interview + focus group + qualitative' was used in searches in PsycINFO and Google Scholar. From a total of 10,106 hits, 7,720 articles were assessed after the removal of duplicates. We also inspected references of relevant studies and searched in online databases and websites.

This search yielded 15 new articles. Based on titles and abstracts, 106 full-text papers were retrieved for screening after initial evaluation of the 7,735 papers. After screening of the 106 full-text papers, 79 papers were deemed relevant for inclusion. Thus, of the 79 papers scrutinized, 50 studies satisfied the following inclusion criteria: (a) studies used qualitative approaches (interviews, focus groups, or case studies) in data collection, (b) studies delineated or described licit and illicit substances used nonmedically by AAS users, and (c) studies were published in English.

We again inspected the characteristics of extracted studies for similarities to curb duplicate extraction and synthesis. The literature search was completed in June 2014. The literature search strategy adhered to Shaw et al.'s [5] recommendations for qualitative literature search as well as the Preferred Reporting Items for Systematic Reviews and Meta-Analyses (PRISMA) guidelines [6]. Figure 1 presents the literature search process.

\section{Data extraction and synthesis}

The first author conducted the study scrutiny and selection. Analysis of the studies was conducted using Smith et al.'s [7] Interpretative Phenomenological Analysis (IPA). Each full-text paper was regarded as a transcript. The first author (DS) read through the full-text papers several times, gaining an overall sense of the themes in the studies through this process. These themes were then highlighted. Using a standardized data extraction form, the first author and another reviewer independently extracted the following data from the included studies: author name and publication year, country, study type, type of AAS users involved in the study, and recruitment site or mode. To assess the quality of the extraction, we calculated inter-reviewer reliability for the two reviewers in SPSS version 20 (IBM Corp.) [8]. DS then independently coded the full-text papers by substance used and reason(s) or motive(s) for use. Study characteristics are presented in Table 1. We have presented all the studies that fall under each substance.

\section{Classification of substances}

We sought to classify the various non-AAS substances used by AAS users into meaningful groups. First, SP provided a functional categorization of the substances. Acknowledging that some AAS users self-administer these substances for purposes contrary to their conventional use, DS built on SP's classification by allocating the substances into SP's groups based on motives for use as presented by users in the literature. For substances for which motive for use was not delineated in the literature, DS grouped them based on Evans-Brown et al.'s [2] classification of human enhancement substances and a classification by the Norwegian Institute of Public Health [59]. JM inspected the grouping and provided further advice. Next, DS allocated substances that at this stage could not be allocated into groups based on the three previous methods by referring to Medscape Drug Reference and Wikipedia [60]. We reached consensus on the classification through further review and discussion. 


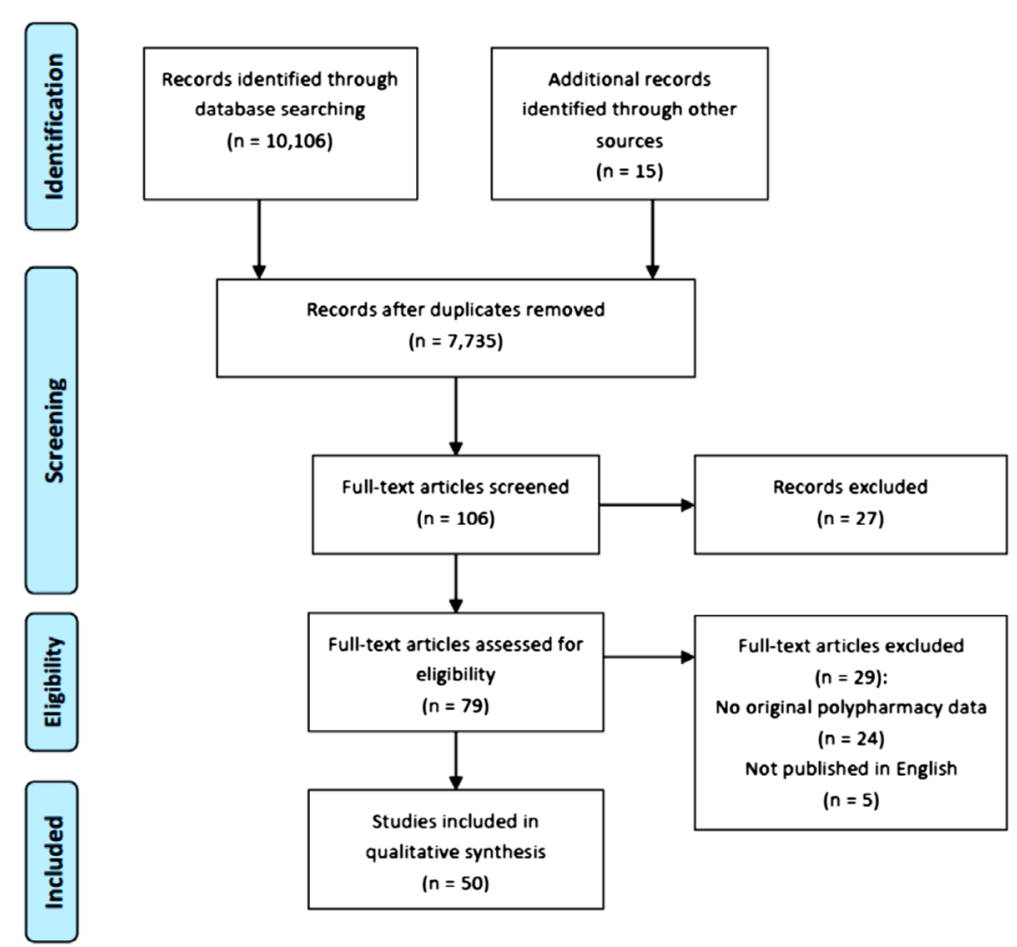

Figure 1 Flow diagram of systematic literature search.

\section{Results and discussion}

\section{Description of studies and inter-reviewer reliability}

A total of 50 studies were included in the metasynthesis. Participants' ages ranged from 14 [34] to 66 years [51]. The year of publication of the studies ranged from 1985 [55] to 2014 [12,13,30]. Studies originated from 10 countries with the highest number from the United States $(n=22)$, followed by Sweden $(n=7)$, England only $(n=5)$, the United Kingdom $(n=4)$, Australia $(n=3)$, and Scotland only $(n=2)$. Additionally, one study originated from Canada, Denmark, France, Iran, and Wales only respectively. One study [16] originated from Australia, Canada, and USA while another described the sample as European [43]. Thirty studies used interviews $[10-12,17,19-22,24,27,28,30-32,34-38,40-43,45,47,48,51,53$, $55,58]$, seven were case studies $[9,23,29,39,49,50,57]$, one used interviews and focus groups [13], and twelve $[14-16,19,25,26,33,44,46,52,54,56]$ used interviews supported by a questionnaire. For the studies that used both interviews and questionnaires, we relied on the qualitative or descriptive results generated from the interviews. There was very good agreement (Kappa $=0.82, p<0.001$ ) between the two reviewers [61]. Through further analysis and dialogue agreement was reached on discrepant extractions.

\section{Substances used prior to AAS initiation}

Before their AAS use debut, some users had experimented with or were regular users of other substances. This was presented by ten studies $[17,21,24,26-29,34,47,53]$. The most prominent of these substances were alcohol, amphetamine, cannabis, and cocaine. Others were analgesics/opioids, heroin, stimulants, and dietary/nutritional supplements such as creatine, and protein powder as well as other unspecified licit and illicit substances (see Table 2).

In Kanayama et al.'s study [26]:

[AAS] users displayed much higher rates of other illicit drug use, abuse, or dependence than non-users, with use of other illicit substances almost always preceding first use of AAS (p. 77).

A recent study by Cornford, Kean, and Nash [13] also highlights heroin use as a precursor to AAS use:

A quick way to make yourself look healthy, isn't it, without being embarrassed about being on heroin [is to use AAS], do you know what I mean. It [heroin] does take a lot of your confidence away don't it and like I say, especially, I lose weight pretty fast when I'm on heroin, do you know what I mean. It [AAS use] is a quick way to just make yourself look healthy again, isn't it (p. 2).

Furthermore, it is important to note that our data also suggested that AAS use may precede the use of other substances for some individuals. In Hoff's study [24]: 
Table 1 Characteristics of qualitative/descriptive studies presenting data on polypharmacy in AAS users

\begin{tabular}{|c|c|c|c|c|c|}
\hline $\begin{array}{l}\text { First author, year, } \\
\text { reference }\end{array}$ & Country & Study type & AAS users & Recruitment site/mode & Non-AAS substances ever used \\
\hline Ahlgrim 2009 [9] & USA & Case study & $\begin{array}{l}\text { 41-year-old male former } \\
\text { bodybuilder }\end{array}$ & Hospital & $\begin{array}{l}\text { Captopril, carvedilol, digoxin, furosemide, growth hormone, } \\
\text { hydrochlorothiazide, spironolactone, torsemide, }\end{array}$ \\
\hline $\begin{array}{l}\text { Angoorani } 2009 \\
{[10]}\end{array}$ & Iran & Interview & $\begin{array}{l}843 \text { bodybuilders aged } 16 \text { to } \\
40 \text { years }\end{array}$ & Gymnasium & Amphetamine \\
\hline Bilard 2011 [11] & France & Interview & 203 bodybuilders & Voluntary & $\begin{array}{l}\text { Beta-2-agonists, cannabinoids, glucocorticosteroids, } \\
\text { peptide hormones }\end{array}$ \\
\hline Chandler 2014 [12] & UK & Interview & 8 persons & $\begin{array}{l}\text { Online forums, syringe } \\
\text { exchange center }\end{array}$ & $\begin{array}{l}\text { Aromatase inhibitors, clenbuterol, 2,4-dinitrophenol, clomiphene, } \\
\text { diuretics, ephedrine, growth hormone releasing peptide, growth } \\
\text { hormone, human chorionic gonadotropin (hCG), insulin- } \\
\text { like growth factor } 1 \text {, insulin, mechano growth factor, melanotan, } \\
\text { mephedrone, tamoxifen, thyroid hormones, viagra } \text { /cialis }^{\oplus}\end{array}$ \\
\hline Cornford 2014 [13] & England & $\begin{array}{l}\text { Interview and } \\
\text { focus group }\end{array}$ & 30 males aged 20 to 40 years & Syringe exchange center & Heroin \\
\hline Davies 2011 [14] & England & $\begin{array}{l}\text { Interview and } \\
\text { questionnaire }^{\dagger}\end{array}$ & 9 male bodybuilders & Gymnasium & Creatine, dietary supplements \\
\hline Dunn 2010 [15] & Australia & $\begin{array}{l}\text { Interview and } \\
\text { questionnaire }^{\dagger}\end{array}$ & 70 persons & Community & $\begin{array}{l}\text { Alcohol, cannabis, cocaine, ecstasy, gamma hydroxybutyrate, } \\
\text { hallucinogens, inhalants, ketamine, amphetamine }\end{array}$ \\
\hline Filiault 2010 [16] & $\begin{array}{l}\text { Australia, Canada, } \\
\text { USA }\end{array}$ & $\begin{array}{l}\text { Interview and } \\
\text { questionnaire }^{\dagger}\end{array}$ & $\begin{array}{l}16 \text { gay male athletes aged } 18 \\
\text { to } 52 \text { years }\end{array}$ & Gay sporting groups & Creatine, dietary supplements, growth hormone, recovery drinks \\
\hline Fudala 2003 [17] & USA & Interview & 7 males aged 22 to 33 years & Gymnasium and community & $\begin{array}{l}\text { Alcohol, analgesics, cannabis, cocaine, stimulants, growth } \\
\text { hormone, human chorionic gonadotropin (hCG), insulin-like } \\
\text { growth factor } 1\end{array}$ \\
\hline Gårevik 2010 [18] & Sweden & Interview & 45 offenders; mean age 30 years & Police station & $\begin{array}{l}\text { Amphetamine, anti-oestrogens, benzodiazepines, cannabis, } \\
\text { clenbuterol, cocaine, diazepam, ephedra, ephedrine, growth } \\
\text { hormone, human chorionic gonadotropin (hCG), heroin, } \\
\text { insulin, sildenafil }\end{array}$ \\
\hline Goldfield 2009 [19] & Canada & $\begin{array}{l}\text { Interview and } \\
\text { questionnaire }^{\dagger}\end{array}$ & 8 female bodybuilders & Gymnasium & Diuretics, laxatives \\
\hline Gruber 1998 [20] & USA & Interview & 19 female weightlifters & Gymnasium & Clenbuterol, ephedrine, narcotics/other drugs \\
\hline Gruber 1999 [21] & USA & Interview & 5 female bodybuilders & Gymnasium & $\begin{array}{l}\text { Alcohol, cannabis, cocaine, clenbuterol, dietary supplements, other } \\
\text { drugs, other performance-enhancing drugs }\end{array}$ \\
\hline Gruber 2000 [22] & USA & Interview & $\begin{array}{l}25 \text { female weightlifters; mean } \\
\text { age } 31 \text { years }\end{array}$ & Gymnasium & $\begin{array}{l}\text { Aminogluthemide, amphetamine, caffeine, clenbuterol, diuretics, } \\
\text { ephedrine, hydroxyl butyrate, human chorionic gonadotropin } \\
\text { (hCG), growth hormone, laxatives, nalbuphine, other opioids, } \\
\text { tamoxifen, thyroid hormones, yohimbine }\end{array}$ \\
\hline Hegazy 2013 [23] & USA & Case study & 28-year-old male & Clinic & Alcohol, amphetamine, opioids \\
\hline Hoff 2012 [24] & Sweden & Interview & $\begin{array}{l}11 \text { male (10 powerlifters, } 1 \\
\text { weightlifter) }\end{array}$ & $\begin{array}{l}\text { Swedish Sports } \\
\text { Confederation }\end{array}$ & Alcohol, amphetamine, cocaine, narcotics, others \\
\hline Hope 2013 [25] & $\begin{array}{l}\text { England and } \\
\text { Wales }\end{array}$ & $\begin{array}{l}\text { Interview and } \\
\text { questionnaire }^{\dagger}\end{array}$ & 340 injecting drug users & Syringe exchange center & $\begin{array}{l}\text { 2,4-dinitrophenol, alcohol, amphetamine, anti-oestrogens, } \\
\text { clenbuterol, cocaine, ephedrine, erythropoietin, growth } \\
\text { hormone, human chorionic gonadotropin (hCG), insulin, }\end{array}$ \\
\hline
\end{tabular}


Table 1 Characteristics of qualitative/descriptive studies presenting data on polypharmacy in AAS users (Continued)

\begin{tabular}{|c|c|c|c|c|}
\hline $\begin{array}{l}\text { Kanayama } 2003 \\
{[26]}\end{array}$ & USA & $\begin{array}{l}\text { Interview and } \\
\text { questionnaire }\end{array}$ & $\begin{array}{l}48 \text { male weightlifters; mean age } \\
29 \text { years }\end{array}$ & $\begin{array}{l}\text { Gymnasium and sports } \\
\text { supplement store }\end{array}$ \\
\hline $\begin{array}{l}\text { Kanayama } 2003 \\
{[27]}\end{array}$ & USA & Interview & $\begin{array}{l}24 \text { male drug users; mean age } \\
32 \text { years }\end{array}$ & Clinic \\
\hline $\begin{array}{l}\text { Kanayama } 2009 \\
{[28]}\end{array}$ & USA & Interview & 62 male weightlifters & $\begin{array}{l}\text { Gymnasium and sports } \\
\text { supplement store }\end{array}$ \\
\hline Katz 1990 [29] & USA & Case study & 23-year-old male bodybuilder & Gymnasium \\
\hline $\begin{array}{l}\text { Kimergård } 2014 \\
{[30]}\end{array}$ & $\begin{array}{l}\text { England and } \\
\text { Wales }\end{array}$ & Interview & $\begin{array}{l}24 \text { males aged } 21 \text { to } 61 \text { years; } \\
\text { mean age } 34 \text { years }\end{array}$ & $\begin{array}{l}\text { Gymnasium, prison, steroid } \\
\text { clinic and charity, syringe } \\
\text { exchange centre }\end{array}$ \\
\hline Klötz 2010 [31] & Sweden & Interview & $\begin{array}{l}33 \text { male prisoners aged } 21 \text { to } \\
52 \text { years }\end{array}$ & Prison \\
\hline
\end{tabular}

Korkia 1993 [32] England, Scotland, Interview and Wales

110 persons (13 female) aged 16 to 63 years

\begin{tabular}{|c|c|c|c|c|}
\hline Korkia 1996 [33] & England & $\begin{array}{l}\text { Interview and } \\
\text { questionnaire }\end{array}$ & 15 females; mean age 28 years & Not specified \\
\hline $\begin{array}{l}\text { Kusserow } 1990 \\
{[34]}\end{array}$ & USA & Interview & $\begin{array}{l}72 \text { ( } 6 \text { female) persons (mostly } \\
\text { adolescents); } 14 \text { to } 25 \text { years; mean } \\
\text { age } 20 \text { years }\end{array}$ & Not specified \\
\hline Larance 2008 [35] & Australia & Interview & 60 males aged 17 to 59 years & $\begin{array}{l}\text { Gymnasium, i } \\
\text { forums, suppl }\end{array}$ \\
\hline Lenehan 1996 [36] & England & Interview & $\begin{array}{l}386 \text { persons aged } 17 \text { to } 56 \text { years; } \\
\text { mean age } 28 \text { years }\end{array}$ & Gymnasium \\
\hline $\begin{array}{l}\text { Lundholm } 2010 \\
\text { [37] }\end{array}$ & Sweden & Interview & 924 (20 female) persons & Prison \\
\hline Malone 1995 [38] & USA & Interview & $\begin{array}{l}77 \text { ( } 6 \text { female) powerlifters and } \\
\text { bodybuilders }\end{array}$ & Gymnasium \\
\hline McBride 1996 [39] & Wales & Case study & $\begin{array}{l}3 \text { males: } 1 \text { AAS dealer and roofer } \\
\text { aged } 27 \text { years, } 1 \text { bodybuilder } \\
\text { aged } 22 \text { years, and } 1 \text { gym owner } \\
\text { aged } 26 \text { years) }\end{array}$ & Not specified \\
\hline
\end{tabular}

melanotan II, nalbuphine, thyroid hormones, diuretics, PDE5 viagra ${ }^{\oplus}$ cialis ${ }^{\oplus}$

Alcohol, cannabis, cocaine, opioids, other narcotics/illicit drugs

Alcohol, cocaine, heroin, nalbuphine, opioids, oxycodone

Alcohol, cannabis, cocaine, opioids, other performanceenhancing drugs, other drugs

Alcohol, cocaine

Amphetamine, clenbuterol, growth hormone, ephedrine, human chorionic gonadotropin (hCG), insulin, melanotan II, sildenafil, tamoxifen

Antidepressants, anti-oestrogen, aspirin ${ }^{\circledast}$, benzodiazepines, caffeine, cannabis, central stimulating drugs, clenbuterol, creatine, diuretics, ephedrine, Gamma hydroxybutyrate, insulin-like growth factor 1, insulin, genotropine, muscle relaxing drugs, myoblast, factor 1, insulin, genotropine, muscle relaxing
opiates, other drugs, potency increasing drugs, somatotropine, testicular function recovering hormones

Antibiotics, corticosteroids, dietary supplements, diuretics, esiclene, human chorionic gonadotropin (hCG), oestrogenantagonist drug, growth hormone, thiomucase, thyroxine

Clenbuterol, growth hormone, nolvadex, nubain ${ }^{\oplus}$ thiomucase, triacana

Alcohol, blood pressure regulators, 'downers', estrogen

inhibitors, growth hormone, cannabis, Recreational substances/drugs, 'uppers'

Anti-oestrogenic agents, aspirin ${ }^{\oplus}$, benzodiazepines, caffeine, cannabis, cocaine, clenbuterol dehydroepiandrosterone (DHEA) diuretics, ecstasy, hallucinogens, heroin, human chorionic gonadotrophin (hCG), ephedrine, growth hormone,

inhalants, insulin-like growth factors, insulin, meth/amphetamine, thyroxine

Clenbuterol, corticosteroids, diuretics, growth hormone, human chorionic gonadotropin (hCG), thyroxine, insulin-like growth factor 1 , nubain ${ }^{\oplus}$, tamoxifen

Benzodiazepines, cannabis, cocaine, meth/amphetamine, opiates

Alcohol, cocaine, hallucinogen, opioids, sedatives, stimulants, tetrahydrocannabinol, tobacco

Amphetamine, cannabis, clenbuterol, human chorionic gonadotropin (hCG), nalbuphine, tamoxifen, temazepam 
Table 1 Characteristics of qualitative/descriptive studies presenting data on polypharmacy in AAS users (Continued)

\begin{tabular}{|c|c|c|c|c|c|}
\hline McKillop 1987 [40] & Scotland & Interview & $\begin{array}{l}8 \text { male bodybuilders aged } 17 \text { to } \\
32 \text { years }\end{array}$ & Gymnasium & $\begin{array}{l}\text { Furosemide, thiazides, thyroxine, human chorionic gonadotropin } \\
\text { (hCG) }\end{array}$ \\
\hline Moss $1992[41]$ & USA & Interview & 50 male bodybuilders & Gymnasium & Clomiphene citrate, human chorionic gonadotropin (hCG) \\
\hline Moss 1993 [42] & USA & Interview & 30 male bodybuilders & Gymnasium & Clomiphene citrate, human chorionic gonadotropin (hCG) \\
\hline Pappa 2012 [43] & Europe & Interview & 9 athletes aged 19 to 26 years & $\begin{array}{l}\text { Community via snowball } \\
\text { sampling }\end{array}$ & $\begin{array}{l}\text { Analgesics, amphetamine, caffeine, cannabis, dietary supplements, } \\
\text { diuretics, erythropoietin. }\end{array}$ \\
\hline Perry $1990[44]$ & USA & $\begin{array}{l}\text { Interview and } \\
\text { questionnaire }^{\dagger}\end{array}$ & $\begin{array}{l}20 \text { male weightlifters aged } 18 \\
\text { to } 28 \text { years }\end{array}$ & Gymnasium & Human chorionic gonadotropin (hCG) \\
\hline Peters 1997 [46] & Australia & $\begin{array}{l}\text { Interview and } \\
\text { questionnaire }^{\dagger}\end{array}$ & $\begin{array}{l}100 \text { persons ( } 6 \text { female) aged } 18 \\
\text { to } 50 \text { years }\end{array}$ & $\begin{array}{l}\text { Advertisements, gymnasium, } \\
\text { interviews, radio, sports shops } \\
\text { and associations, syringe } \\
\text { exchange centre }\end{array}$ & $\begin{array}{l}\text { Alcohol, aminogluthimide, amphetamine, antibiotics, beta } \\
\text { blockers, caffeine, cannabis, chromium picolinate, clenbuterol, } \\
\text { cocaine, daoni }{ }^{\oplus} \text {, dietary supplement, diuretics, ecstasy, ephedrine, } \\
\text { growth hormone, human chorionic gonadotropin (hCG), } \\
\text { hydroxocobal amin, insulin-like growth factor 1, insulin, oestrogen } \\
\text { antagonist, pregny }{ }^{\oplus} \text {, proviron }{ }^{\oplus} \text {, teroxin (T3), thyroxine }\end{array}$ \\
\hline Pope 1988 [47] & USA & Interview & $\begin{array}{l}41 \text { male bodybuilders and } \\
\text { footballers }\end{array}$ & Gymnasium & $\begin{array}{l}\text { Alcohol, cannabis, cigarettes, cocaine, human chorionic } \\
\text { gonadotropin (hCG) }\end{array}$ \\
\hline Rashid 2000 [49] & USA & Case study & 40-year-old male & Clinic & $\begin{array}{l}\text { Cocaine, cannabis, 'uppers', 'downers', lysergic acid diethylamide } \\
\text { (LSD) }\end{array}$ \\
\hline Schäfer 2011 [50] & Denmark & Case study & 26-year-old male bodybuilder & Clinic & Erythropoietin \\
\hline Silvester 1995 [51] & USA & Interview & $\begin{array}{l}22 \text { former athletes aged } 36 \text { to } \\
66 \text { years }\end{array}$ & Not specified & Growth hormone \\
\hline Skårberg 2007 [52] & Sweden & $\begin{array}{l}\text { Interview and } \\
\text { questionnaire }^{\dagger}\end{array}$ & $\begin{array}{l}18 \text { male drug users; mean age } \\
35 \text { years }\end{array}$ & Clinic & Alcohol, narcotics/other drugs \\
\hline Skårberg 2008 [53] & Sweden & Interview & 6 drug users (2 female) & Clinic & $\begin{array}{l}\text { Alcohol, amphetamine, analgesics, anti-catabolics, anti-oestrogens, } \\
\text { aspirin }{ }^{\oplus} \text {, benzodiazepines, bronchodilators, buprenorphine, } \\
\text { caffeine, cannabis, cocaine, codeine, conjugated linoleic acid, } \\
\text { creatine, dietary supplements, ecstasy, ephedra, ephedrine, growth } \\
\text { hormone, Herbal products, insulin growth factor 1, insulin, protein } \\
\text { powder, testosterone releasers }\end{array}$ \\
\hline Skårberg 2009 [54] & Sweden & $\begin{array}{l}\text { Interview and } \\
\text { questionnaire }^{\dagger}\end{array}$ & 32 male drug users & Clinic & $\begin{array}{l}\text { Alcohol, amphetamine, anti-oestrogen (clomid), analgesics, } \\
\text { anti-acne drug, anti-catabolics, anti-depressants, anti-hypertensive } \\
\text { drugs, anti-oestrogens, benzodiazepines, bronchodilators, cannabis, } \\
\text { cocaine, creatine, dietary supplements, diuretics, ephedrine, fat-loss } \\
\text { agents, gamma hydroxybutyrate, growth hormone, heroin, insulin, } \\
\text { insulin-like growth factor 1, levodopa, muscle oil (synthol), } \\
\text { non-steroidal anti-inflammatory drugs, opioid, plant steroid } \\
\text { compounds, protein powder, stimulants, testosterone boosters, } \\
\text { thyroid hormone }\end{array}$ \\
\hline Strauss 1985 [55] & USA & Interview & $\begin{array}{l}10 \text { weight-trained female } \\
\text { athletes; mean age } 33 \text { years }\end{array}$ & Personal contact & $\begin{array}{l}\text { Acetaminophen, aspirin }{ }^{\oplus} \text {, benoxaprofen, Ben-Gay }{ }^{\oplus} \text {, caffeine, } \\
\text { calcium, choline and inositol, dietary supplements, dimethyl }\end{array}$ \\
\hline
\end{tabular}


Table 1 Characteristics of qualitative/descriptive studies presenting data on polypharmacy in AAS users (Continued)

\begin{tabular}{|c|c|c|c|c|c|}
\hline & & & & & $\begin{array}{l}\text { sulfoxide, codeine, electrolyte solution, epinephrine, furosemide, } \\
\text { growth hormone, levodopa, lidocaine, naproxen, oxycodone } \\
\text { hydrochloride, phenylbutazone, piroxicam, potassium, suntan } \\
\text { pills, thyroglobulin, vitamins }\end{array}$ \\
\hline Tallon 2007 [56] & Scotland & $\begin{array}{l}\text { Interview and } \\
\text { questionnaire }\end{array}$ & $\begin{array}{l}30 \text { males aged } 18 \text { to } 43 \text { years; } \\
\text { mean age } 27 \text { years }\end{array}$ & Gymnasium & $\begin{array}{l}\text { Alcohol, cannabis, cocaine, clenbuterol, dietary supplements, } \\
\text { diuretics, ecstasy, growth hormone, insulin, tamoxifen }\end{array}$ \\
\hline $\begin{array}{l}\text { Wilson-Fearon } \\
1999[57]\end{array}$ & England & Case study & 29-year-old bodybuilder & Not specified & $\begin{array}{l}\text { Clenbuterol, dietary supplements, diuretics, growth hormone, } \\
\text { human chorionic gonadotropin (hCG), thiomucase }\end{array}$ \\
\hline Wines 1999 [58] & USA & Interview & $\begin{array}{l}11 \text { weightlifters ( } 5 \text { female) aged } \\
19 \text { to } 42 \text { years }\end{array}$ & Gymnasium & $\begin{array}{l}\text { Alcohol, buprenorphine, heroin, hydrocodone, nalbuphine, other } \\
\text { drugs }\end{array}$ \\
\hline
\end{tabular}

We relied on the qualitative results generated from the interview. 
Table 2 Non-AAS substances used before AAS use debut, reason(s)/motive(s) for use, and studies

\begin{tabular}{|c|c|c|}
\hline Substance & Reason(s) for use & Studies (First author, reference) \\
\hline Alcohol & Better sleep and relaxation & $\begin{array}{l}\text { Fudala [17]; Gruber [21]; Hoff [24]; Kanayama [26]; Katz [29]; } \\
\text { Kusserow [34]; Perry [47]; Skårberg [53] }\end{array}$ \\
\hline Amphetamine & $\begin{array}{l}\text { Boosting training, alertness, } \\
\text { psychological wellbeing }\end{array}$ & Hoff [24]; Skårberg [53] \\
\hline Analgesics/opioids $^{\dagger}$ & Pain relief & Kanayama [26] \\
\hline Cannabis & NS & Fudala [17]; Hoff [24]; Kanayama [26]; Kusserow [34]; Perry [47] \\
\hline Cocaine & NS & Fudala [17]; Gruber [21]; Kanayama [26]; Katz [29]; Perry [47] \\
\hline Creatine & Boosting training & Skårberg [53] \\
\hline Dietary/nutritional supplements ${ }^{\dagger}$ & Boosting training, energy & Skårberg [53] \\
\hline Heroin & NS & Cornford [13] \\
\hline Protein powder & Boosting training & Skårberg [53] \\
\hline Stimulants $^{\dagger}$ & NS & Kusserow [34] \\
\hline Other IPEDs, licit and illicit substances & NS & Gruber [21]; Hoff [24]; Kanayama [27,28]; Perry [47] \\
\hline
\end{tabular}

IPEDs: Image and performance-enhancing drugs.

NS: Not specified.

${ }^{\dagger}$ Substance class - not specified.

Respondent 8 reported using narcotics after he had started using AAS. In this case, alcohol and drug abuse cannot explain why he started doping [using AAS]. However, AAS use seems to have led him into drug use and criminality in order to finance his extensive AAS use and investment in elite powerlifting. This respondent became aggressive and violent when he combined AAS and alcohol. Due to these side effects he changed from alcohol to cocaine as his primary social drug when he was on AAS (p. 63).

\section{Use of supplementary/ancillary substances}

AAS users often engaged in stacking and the use of various licit and illicit substances during their 'on cycles' as previously shown. For instance, in a study by McBride [39], “...Mr B had initially used nalbuphine in conjunction with anabolic steroids, clenbuterol, ephedrine, and tamoxifen, all to aid bodybuilding" (p. 69). Indeed, in a study [46] of 100 AAS users: "A number of other drugs were used in addition to AAS as part of their training routine by $49 \%$ of the sample" (p. 49).

The most popular supplementary/ancillary substances declared by AAS users in multiple studies were: alcohol, amphetamine/meth, aspirin ${ }^{\oplus}$ caffeine, cannabis/cannabinoids, clenbuterol, clomiphene citrate, cocaine, codeine, creatine, ephedra/ephedrine, erythropoietin, furosemide, gamma hydroxybutyrate (GHB), growth hormone, heroin, human chorionic gonadotropin (hCG), insulin, insulin-like growth factor 1 (IGF-1), melanotan, nalbuphine/nubain', protein powder, tamoxifen, thyroxine, and tobacco. Other popular classes of substances presented were analgesics/opioids, anti-oestrogens, benzodiazepines, dietary/nutritional supplements, diuretics, hallucinogens, and stimulants (see Table 3).

\section{Lifetime polypharmacy}

We also investigated lifetime use of other substances by AAS users. The most popular substances (declared in multiple studies) were: 2,4-dinitrophenol (DNP), alcohol, aminogluthimide, amphetamine/meth, aspirin ${ }^{\oplus}$, buprenorphine, caffeine, cannabis/cannabinoids, clenbuterol, clomiphene citrate, cocaine, codeine, creatine, ephedra/ephedrine, erythropoietin (EPO), furosemide, gamma hydroxybutyrate (GHB), growth hormone, heroin, human chorionic gonadotropin (hCG), insulin, insulin-like growth factor 1 (IGF-1), ketamine, levodopa, lysergic acid diethylamide (LSD), melanotan, nalbuphine/nubain ${ }^{\oplus}$, oxycodone, protein powder, sildenafil/viagra ${ }^{\circ} /$ cialis $^{\oplus}$, tamoxifen, thiomucase, thyroxine, and yohimbine. Other popular classes of substances presented were analgesics/opioids, antibiotics, anti-catabolics, anti-oestrogens, benzodiazepines, blood pressure regulators, bronchodilators, dietary/nutritional supplements, diuretics, hallucinogens, inhalants, stimulants, and testosterone releasers/boosters.

Of the above substances, the most commonly identified in studies include alcohol, cannabis/cannabinoids, cocaine, growth hormone, human chorionic gonadotropin (hCG), amphetamine/meth, clenbuterol, ephedra/ephedrine, insulin, and thyroxine. Commonly identified classes of substances include analgesics/opioids, dietary/nutritional supplements, diuretics, and anti-oestrogens (see Table 3).

\section{Groups of non-AAS substances used by AAS users}

Our classification of the various substances used by AAS users resulted in 13 main groups: analgesics/non-steroidal anti-inflammatory drugs/opioids, anti-oestrogens, cardiovascular drugs, central nervous system depressants, central nervous system stimulants, cosmetic drugs, dietary/ 
nutritional supplements, diuretics, fat burning/weight loss drugs, muscle/strength-enhancement hormones, non-hormone muscle/strength-enhancement drugs, recreational substances/drugs, and sexual enhancement drugs (see Table 4). These groups of substances are briefly discussed below.

\section{Analgesics/non-steroidal anti-inflammatory drugs/opioids} These drugs include aspirin ${ }^{\oplus}$, codeine, and oxycodone. This group of drugs was used for relieving inflammation, pain, and fever emanating from exercise, sports participation or the recreational and occupational activities of AAS users.

\section{Anti-oestrogens}

Anti-oestrogens include aminogluthimide, clomiphene, and tamoxifen. These drugs were used for reducing the oestrogen-like side effects of AAS use such as preventing gynecomastia. They were also used for endurance, improved testosterone production, and burning body fat.

\section{Cardiovascular drugs}

These drugs such as captopril, carvedilol, and digoxin were used for improved functioning of the cardiovascular system such as lowering blood pressure and reducing the risk of myocardial infarction, as well as burning body fat.

\section{Central nervous system depressants}

Examples of depressants are buprenorphine, hydrocodone, and oxycodone. The purposes for which these drugs were used were improved sleep, relaxation, and elevation of mood.

\section{Central nervous system stimulants}

Stimulants including epinephrine, amphetamine/methamphetamine, and yohimbine were used for alertness, boosting training, burning body fat, increased aggression and strength (including sexual), and psychological wellbeing.

\section{Cosmetic drugs}

Cosmetic or aesthetic drugs such as esiclene, melanotan II, and thiomucase were used in order to deal with acne, and for: inflammatory effects on smaller muscles, skin tanning, and a leaner physique thus enhancing physical appearance.

\section{Dietary/nutritional supplements}

These supplements such as calcium, glutamine, and potassium were consumed to provide essential nutrients to supplement the diet and combat the risk of illness.

\section{Diuretics}

Diuretics such as furosemide, hydrochlorothiazide, and spironolactone were used for combating side effects of AAS use such as water retention, together with masking the use of AAS and other doping agents.

\section{Fat burning/weight loss drugs}

These drugs include 2,4-dinitrophenol (DNP), conjugated linoleic acid, and teroxin (T3) and were used for suppression of appetite, increased metabolism, and reduced absorption of body fat as a means to burning body fat and losing weight.

\section{Muscle/strength-enhancement substances}

Two types of muscle/strength-enhancement substances were presented in the literature: hormones and nonhormones. Examples of muscle/strength-enhancement hormones are growth hormones, growth hormone releasing peptide (GHRP), and insulin. Non-hormone muscle/strength-enhancement drugs include clenbuterol used by some in an attempt to enhance the size and structure of muscles, as well as boosting strength.

\section{Recreational substances/drugs}

Recreational substances/drugs such as cannabis/cannabinoids, cocaine, and lysergic acid diethylamide (LSD) were used to alter experiences, elevate mood, and create psychological wellbeing as well as for relaxation.

\section{Sexual enhancement drugs}

These drugs such as phosphodiesterase-5 inhibitors (PDE5i), melanotan II, and sildenafil were used for dealing with testicular atrophy, improved sexual desire or arousal as well as erectile functioning.

In sum, the above groups of substances were used to enhance the effects of AAS, combat the side effects of AAS, and for recreational or relaxation purposes, as well as sexual enhancement. It is important to note that there is overlap between some of the groups. For instance, some central nervous system depressants may be misused for promoting sleep as well as their analgesic properties. Again, some muscle/strength-enhancement hormones are used for direct muscle enhancing properties and others for counteracting shutdown of endogenous testosterone production. Additionally, some of the substances are used for multiple purposes. For instance, melanotan II is used for tanning the skin and also as self-treatment for erectile dysfunction resulting from long-term AAS use. Others may use melanotan II to self-treat specific conditions such as rosacea or fibromyalgia and others may use melanotan for the self-reported weight loss effects due to appetite suppression. It is also important to note that some of the alleged properties or uses are not scientifically well documented such as the use of insulin for burning body fat [54]. Furthermore, the quality, safety, and efficacy of substances obtained from the illicit market cannot be known, with adulteration usually commonplace $[2,63]$. 
Table 3 Use of non-AAS substances, reason(s)/motive(s) for use, and studies

\begin{tabular}{|c|c|c|c|c|c|}
\hline \multirow[b]{2}{*}{ Substance } & \multicolumn{2}{|c|}{ Current polypharmacy (Combined with AAS) } & \multicolumn{2}{|l|}{ Lifetime polypharmacy (Ever use) } & \multirow[b]{2}{*}{$\begin{array}{l}\text { Number of } \\
\text { studies }\end{array}$} \\
\hline & Reason(s) for use & $\begin{array}{l}\text { Studies (First } \\
\text { author, reference) }\end{array}$ & Reason(s) for use & $\begin{array}{l}\text { Studies (First } \\
\text { author, reference) }\end{array}$ & \\
\hline 2,4-dinitrophenol & NS & Chandler [12] & NS & $\begin{array}{l}\text { Chandler [12]; Dunn [15]; Hope [25]; Larance } \\
\text { [35] }\end{array}$ & 4 \\
\hline Acetaminophen & NS & Strauss [55] & NS & Strauss [55] & 1 \\
\hline Alcohol & Better sleep and relaxation & $\begin{array}{l}\text { Chandler [12]; Hegazy [23]; } \\
\text { Kanayama [28]; Kusserow [34]; } \\
\text { Lundholm [37]; Malone [38]; } \\
\text { Peters [46]; Perry [48]; Skårberg } \\
\text { [52-54] }\end{array}$ & Better sleep and relaxation & $\begin{array}{l}\text { Chandler [12]; Dunn [15]; Fudala [17]; Gruber } \\
\text { [21,22]; } \\
\text { Hegazy [23]; Hoff [24]; Hope [25]; Kanayama } \\
\text { [26-28]; } \\
\text { Katz [29]; Kusserow [34]; Malone [38]; } \\
\text { Peters [46]; } \\
\text { Perry [48]; Skårberg [52-54]; Tallon [56]; } \\
\text { Wines [58] }\end{array}$ & 23 \\
\hline Aminogluthimide & $\begin{array}{l}\text { Reducing receptors' attraction } \\
\text { to cortisol }\end{array}$ & Peters [46] & $\begin{array}{l}\text { Reducing receptors' attraction } \\
\text { to cortisol }\end{array}$ & Gruber [22]; Peters [46] & 2 \\
\hline Amyl nitrate & NS & Chandler [12] & NS & Chandler [12] & 1 \\
\hline Analgesics/opioids ${ }^{\dagger}$ & Pain relief & $\begin{array}{l}\text { Ahlgrim [9]; Hegazy [23]; } \\
\text { Kanayama [28]; Klötz [31]; } \\
\text { Kusserow [34]; Lundholm [37]; } \\
\text { Malone [38]; McBride [39]; } \\
\text { Pappa [43]; Skårberg [53] }\end{array}$ & Pain relief & $\begin{array}{l}\text { Ahlgrim [9]; Fudala [17]; Gruber [22]; } \\
\text { Hegazy [23]; } \\
\text { Kanayama [26,28], Klötz [31]; Kusserow } \\
\text { [34]; Malone } \\
\text { [38]; McBride [39]; Pappa [43]; Rashid [49]; } \\
\text { Skårberg } \\
\text { [53,54] }\end{array}$ & 14 \\
\hline Anti-acne drugs ${ }^{\dagger}$ & - & - & Combating acne & Skårberg [54] & 1 \\
\hline Antibiotics $^{\dagger}$ & Combating acne & Peters [46] & Combating acne & Korkia [32]; Peters [46] & 2 \\
\hline Anti-catabolics $^{\dagger}$ & NS & Skårberg [53] & $\begin{array}{l}\text { Facilitating synthesis of hepatic } \\
\text { protein and nitrogen economy }\end{array}$ & Skårberg [53,54] & 2 \\
\hline Anti-depressants $^{\dagger}$ & Combating side effects & Klötz [31] & $\begin{array}{l}\text { Combating side effects, depression } \\
\text { relief, boosting levels of serotonin } \\
\text { and noradrenaline }\end{array}$ & Klötz [31]; Skårberg [54] & 2 \\
\hline Anti-oestrogens $^{\dagger}$ & $\begin{array}{l}\text { Burning fat, combating } \\
\text { gynecomastia, } \\
\text { reducing effects on oestrogen }\end{array}$ & $\begin{array}{l}\text { Klötz [31]; Kusserow [34]; } \\
\text { Peters [46]; Skårberg [53] }\end{array}$ & $\begin{array}{l}\text { Combating gynecomastia, burning } \\
\text { fat, reducing effects on oestrogen }\end{array}$ & $\begin{array}{l}\text { Gårevik [18]; Hope [25]; Klötz [31]; } \\
\text { Korkia [32]; } \\
\text { Kusserow [34]; Larance [35]; Peters [46]; } \\
\text { Skårberg } \\
{[53,54]}\end{array}$ & 9 \\
\hline Aromatase inhibitors $^{\dagger}$ & NS & Chandler [12] & NS & Chandler [12] & 1 \\
\hline Aspirin ${ }^{\oplus}$ & NS & $\begin{array}{l}\text { Klötz [31]; Perry [45]; Skårberg } \\
\text { [53]; Strauss [55] }\end{array}$ & NS & $\begin{array}{l}\text { Klötz [31]; Larance [35]; Perry [45]; } \\
\text { Skårberg [53]; } \\
\text { Strauss [55] }\end{array}$ & 5 \\
\hline Ben-Gay ${ }^{\oplus}$ & NS & Strauss [55] & NS & Strauss [55] & 1 \\
\hline
\end{tabular}


Table 3 Use of non-AAS substances, reason(s)/motive(s) for use, and studies (Continued)

\begin{tabular}{|c|c|c|c|}
\hline Benoxaprofen & NS & Strauss [55] & NS \\
\hline Benzodiazepines $^{\dagger}$ & $\begin{array}{l}\text { Better sleep, combating } \\
\text { side effects, } \\
\text { relaxation }\end{array}$ & $\begin{array}{l}\text { Klötz [31]; Larance [35]; } \\
\text { Lundholm [37]; McBride [39]; } \\
\text { Skårberg [53] }\end{array}$ & $\begin{array}{l}\text { Combating side effects, enhancin } \\
\text { sleep and relaxation, self-control, } \\
\text { sedation }\end{array}$ \\
\hline Beta blockers ${ }^{\dagger}$ & Burning fat & Peters [46] & Burning fat \\
\hline Beta-2-agonists $^{\dagger}$ & - & - & NS \\
\hline $\begin{array}{l}\text { Blood pressure } \\
\text { regulators }^{\dagger}\end{array}$ & NS & Kusserow [34] & Lower blood pressure \\
\hline Bronchodilators $^{\dagger}$ & Energy and boosting training & Skårberg [53] & $\begin{array}{l}\text { Burning fat, energy and boosting } \\
\text { training, increasing strength }\end{array}$ \\
\hline Buprenorphine & NS & Skårberg [53] & NS \\
\hline Caffeine & Burning fat & $\begin{array}{l}\text { Klötz [31]; Pappa [43]; Perry } \\
\text { [45]; Peters [46]; Skårberg [53]; } \\
\text { Strauss [55] }\end{array}$ & Burning fat \\
\hline Calcium & - & - & NS \\
\hline Cannabis/cannabinoids & Enhancing sleep, relaxation & $\begin{array}{l}\text { Chandler [12]; Kanayama } \\
\text { [27,28]; Klötz [31]; Kusserow } \\
\text { [34]; Lundholm [37]; Malone } \\
\text { [38]; McBride [39]; Pappa [43]; } \\
\text { Peters [46]; Perry [48]; Skårberg } \\
\text { [53,54] }\end{array}$ & Enhancing sleep, relaxation \\
\hline Captopril & NS & Ahlgrim [9] & NS \\
\hline Carvedilol & NS & Ahlgrim [9] & NS \\
\hline Choline and inositol & NS & Strauss [55] & NS \\
\hline Chromium picolinate & Reducing body weight & Peters [46] & Reducing body weight \\
\hline Clenbuterol & $\begin{array}{l}\text { Anabolic effects, burning fat, } \\
\text { removing skin fluid, weight loss }\end{array}$ & $\begin{array}{l}\text { Chandler [12]; Gruber [21]; } \\
\text { Kimergård [30]; Klötz [31]; } \\
\text { Lenehan [36]; McBride [39]; } \\
\text { Peters [46]; Wilson-Fearon [57] }\end{array}$ & $\begin{array}{l}\text { Anabolic effects, burning fat, } \\
\text { removing skin fluid, weight loss }\end{array}$ \\
\hline Clomiphene citrate & NS & $\begin{array}{l}\text { Chandler [12]; Moss [41,42]; } \\
\text { Perry [45] }\end{array}$ & NS \\
\hline Cocaine & $\begin{array}{l}\text { Boosting training, burning fat, } \\
\text { increasing strength }\end{array}$ & $\begin{array}{l}\text { Chandler [12]; Kanayama [28]; } \\
\text { Larance [35]; Lundholm [37]; } \\
\text { Malone [38]; Peters [46]; } \\
\text { Skårberg }[53,54]\end{array}$ & $\begin{array}{l}\text { Boosting training, burning fat, } \\
\text { increasing strength }\end{array}$ \\
\hline Codeine & Boosting training & Skårberg [53]; Strauss [55] & Boosting training \\
\hline Conjugated linoleic acid & Burning fat & Skårberg [53] & Burning fat \\
\hline
\end{tabular}

Strauss [55]

(3) 8

[37]; Malone [38]; McBride

[39]; Skårberg [53,54]

Peters [46]

Bilard [11]

Kusserow [34]; Skårberg [54]

Skårberg [53,54]

Skårberg [53]; Wines [58]

Gruber [22]; Klötz [31]; Larance [35]; Pappa [43]; Peters [46]; Perry [45];

Skårberg [53]; Strauss [55]

Strauss [55]

Bilard [11]; Chandler [12]; Dunn [15];

Fudala [17]; Gruber [21,22]; Hoff [24];

Kanayama [27,28]; Klötz [31]; Kusserow

[34]; Larance [35]; Malone [38]; McBride

[39]; Pappa [43]; Peters [46]; Perry [48];

Rashid [49]; Skårberg [53,54]; Tallon [56]

Ahlgrim [9]

Ahlgrim [9]

Strauss [55]

Peters [46]

Chandler [12]; Gruber [20-22]; Hope [25]; Kimergård [30]; Klötz [31]; Korkia [33]; Larance [35]; Lenehan [36]; McBride [39]; Peters [46]; Tallon [56]; Wilson-Fearon [57]

Chandler [12]; Moss [41,42]; Perry [45]

Chandler [12]; Dunn [15]; Fudala [17]; Gårevik [18]; Gruber [21]; Hoff [24]; Hope [25]; Kanayama [26-28]; Katz [29]; Larance [35]; Lundholm [37]; Malone [38]; Peters [46]; Rashid [49]; Skårberg [53,54]; Tallon

Skårberg [53]; Strauss [55] 
Table 3 Use of non-AAS substances, reason(s)/motive(s) for use, and studies (Continued)

\begin{tabular}{|c|c|c|c|c|c|}
\hline Corticosteroids $^{\dagger}$ & NS & Lenehan [36] & NS & Bilard [1 1]; Korkia [32]; Lenehan [36] & 3 \\
\hline Creatine & Enhancing the effects of training & $\begin{array}{l}\text { Klötz [31]; Skårberg [53]; Perry } \\
\text { [45] }\end{array}$ & $\begin{array}{l}\text { Anabolic effects, endurance } \\
\text { booster, } \\
\text { enhancing the effects of training, } \\
\text { recovery aid }\end{array}$ & $\begin{array}{l}\text { Davies [14]; Filiault [16]; Hoff [24]; } \\
\text { Klötz [31]; Perry [45]; Skårberg [53,54] }\end{array}$ & 7 \\
\hline Daonil ${ }^{\oplus}$ & Increasing insulin release & Peters [46] & Increasing insulin release & Peters [46] & 1 \\
\hline $\begin{array}{l}\text { Dehydroepiandrosterone } \\
\text { (DHEA) }\end{array}$ & - & - & NS & Larance [35] & 1 \\
\hline Diazepam & - & - & NS & Gårevik [18] & 1 \\
\hline $\begin{array}{l}\text { Dietary/nutritional } \\
\text { supplements }^{\dagger}\end{array}$ & $\begin{array}{l}\text { Energy and boosting training, } \\
\text { nutrition }\end{array}$ & $\begin{array}{l}\text { Pappa [43]; Perry [45]; Peters } \\
\text { [46]; Skårberg [53,54]; Strauss } \\
\text { [55]; Wilson-Fearon [57] }\end{array}$ & $\begin{array}{l}\text { Energy and boosting training, anabolic } \\
\text { effects, endurance booster, nutrition, } \\
\text { recovery aid }\end{array}$ & $\begin{array}{l}\text { Davies [14]; Filiault [16]; Gruber [21]; Korkia } \\
\text { [32]; Pappa [43]; Perry [45]; Peters [46]; } \\
\text { Skårberg [53,54]; Strauss [55]; Tallon [56]; } \\
\text { Wilson-Fearon [57] }\end{array}$ & 12 \\
\hline Digoxin & NS & Ahlgrim [9] & NS & Ahlgrim [9] & 1 \\
\hline Dimethyl sulfoxide & NS & Strauss [55] & NS & Strauss [55] & 1 \\
\hline Diuretics $^{\dagger}$ & $\begin{array}{l}\text { Combating side effects, masking } \\
\text { doping drugs, reducing fluid levels }\end{array}$ & $\begin{array}{l}\text { Chandler [12]; Klötz [31]; } \\
\text { Lenehan [36]; Pappa [43]; } \\
\text { Peters [46]; Wilson-Fearon [57] }\end{array}$ & $\begin{array}{l}\text { Combating side effects, masking doping } \\
\text { drugs, reducing fluid levels }\end{array}$ & $\begin{array}{l}\text { Chandler [12]; Goldfield [19]; Hope [25]; Klötz } \\
\text { [31]; Korkia [32]; Larance [35]; Lenehan [36]; } \\
\text { Pappa [43]; Peters [46]; Skårberg [54]; Tallon } \\
\text { [56]; Wilson-Fearon [57] }\end{array}$ & 12 \\
\hline Electrolyte solution & NS & Strauss [55] & NS & Strauss [55] & 1 \\
\hline Ephedra/Ephedrine & $\begin{array}{l}\text { Energy and boosting training, } \\
\text { enhancing weight loss }\end{array}$ & $\begin{array}{l}\text { Chandler [12]; Kimergård [30]; } \\
\text { Klötz [31]; McBride [39]; Perry } \\
\text { [45]; Peters [46]; Skårberg [53] }\end{array}$ & $\begin{array}{l}\text { Energy and boosting training, burning } \\
\text { fat, enhancing weight loss, increasing } \\
\text { strength }\end{array}$ & $\begin{array}{l}\text { Chandler [12]; Gårevik [18]; Gruber [20]; Gårevik } \\
\text { [18]; Hope [25]; Kimergård [30]; Klötz [31]; } \\
\text { Larance } \\
\text { [35]; McBride [39]; Perry [45]; Peters [46]; } \\
\text { Skårberg } \\
\text { [53,54] }\end{array}$ & 14 \\
\hline Epinephrine & NS & Strauss [55] & NS & Strauss [55] & 1 \\
\hline Erythropoietin (EPO) & NS & Pappa [43]; Schäfer [50] & NS & Hope [25]; Pappa [43]; Schäfer [50] & 3 \\
\hline Esiclene & - & - & NS & Korkia [32] & 1 \\
\hline Fat-loss agents ${ }^{\dagger}$ & - & - & Burning fat & Skårberg [54] & 1 \\
\hline Furosemide & Weight loss & Ahlgrim [9]; Strauss [55] & Weight loss & Ahlgrim [9]; McKillop [40]; Strauss [55] & 3 \\
\hline $\begin{array}{l}\text { Gamma hydroxybutyrate } \\
\text { (GHB) }\end{array}$ & Enhancing sleep & $\begin{array}{l}\text { Chandler [12]; Klötz [31]; } \\
\text { Skårberg [54] }\end{array}$ & Enhancing sleep & $\begin{array}{l}\text { Chandler [12]; Dunn [15]; Gruber [22]; } \\
\text { Klötz [31]; Skårberg [54] }\end{array}$ & 5 \\
\hline Genotropine & NS & Klötz [31] & NS & Klötz [31] & 1 \\
\hline Glutamine & NS & Perry [45] & NS & Perry [45] & 1 \\
\hline Growth hormone & $\begin{array}{l}\text { Anabolic effects and strength, } \\
\text { burning fat, weight loss }\end{array}$ & $\begin{array}{l}\text { Chandler [12]; Kimergård [30]; } \\
\text { Kusserow [34]; Lenehan [36]; } \\
\text { Peters [46]; Skårberg [53,54]; } \\
\text { Strauss [55]; Wilson-Fearon [57] }\end{array}$ & $\begin{array}{l}\text { Anabolic effects, burning fat, } \\
\text { endurance } \\
\text { booster, recovery aid, weight loss }\end{array}$ & $\begin{array}{l}\text { Chandler [12]; Filiault [16]; Fudala [17]; } \\
\text { Gårevik [18]; } \\
\text { Hope [25]; Kimergård [30]; Korkia [32,33]; } \\
\text { Kusserow }\end{array}$ & 18 \\
\hline
\end{tabular}




\begin{tabular}{|c|c|c|c|c|}
\hline & & & & $\begin{array}{l}\text { [34]; Larance [35]; Lenehan [36]; Peters } \\
\text { [46]; Silvester } \\
\text { [51]; Skårberg [53,54]; Strauss [55]; Tallon [56]; } \\
\text { Wilson-Fearon [57] }\end{array}$ \\
\hline $\begin{array}{l}\text { Growth hormone } \\
\text { releasing peptides }\end{array}$ & NS & Chandler [12] & NS & Chandler [12] \\
\hline Hallucinogens $^{\dagger}$ & NS & Larance [35]; Malone [38] & NS & Dunn [15]; Larance [35]; Malone [38] \\
\hline Herbal products $^{\dagger}$ & NS & Skårberg [53] & Increasing strength & Skårberg [53,54] \\
\hline Heroin & Enhancing sleep, pain relief & $\begin{array}{l}\text { Cornford [13]; Larance [35]; } \\
\text { Skårberg [54] }\end{array}$ & Enhancing sleep, pain relief & $\begin{array}{l}\text { Gårevik [18]; Cornford [13]; Kanayama [27]; } \\
\text { Larance } \\
\text { [35]; Skårberg [54]; Wines [58] }\end{array}$ \\
\hline $\begin{array}{l}\text { Human chorionic } \\
\text { gonadotropin (hCG) }\end{array}$ & $\begin{array}{l}\text { Minimizing depressive symptoms } \\
\text { upon AAS cessation/withdrawal, } \\
\text { improving testosterone production, } \\
\text { preventing weight loss, stopping } \\
\text { testicular atrophy, increasing } \\
\text { strength }\end{array}$ & $\begin{array}{l}\text { Chandler [12]; Kimergård [30]; } \\
\text { Lenehan [36]; McBride [39]; } \\
\text { Moss [41,42]; Perry [44]; Peters } \\
\text { [46]; Perry [47]; Korkia [32]; } \\
\text { Wilson-Fearon [57] }\end{array}$ & $\begin{array}{l}\text { Anabolic effects, increasing } \\
\text { testosterone production, minimizing } \\
\text { depressive symptoms upon AAS } \\
\text { cessation/withdrawal, } \\
\text { preventing weight loss, } \\
\text { stopping testicular atrophy, } \\
\text { increasing } \\
\text { strength }\end{array}$ & $\begin{array}{l}\text { Chandler [12]; Fudala [17]; Gruber [22]; } \\
\text { Gårevik [18]; } \\
\text { Hope [25]; Kimergård [30]; Korkia [32]; } \\
\text { Larance [35]; } \\
\text { Lenehan [36]; McBride [39]; McKillop } \\
\text { [40]; Moss } \\
\text { [41,42]; Perry [44]; Peters [46]; Perry [47]; } \\
\text { Wilson-Fearon [57] }\end{array}$ \\
\hline Hydrochlorothiazide & Weight loss & Ahlgrim [9] & Weight loss & Ahlgrim [62] \\
\hline Hydrocodone & - & - & NS & Wines [58] \\
\hline Hydroxocobal amin & Weight gain & Peters [46] & Weight gain & Peters [46] \\
\hline Inhalants ${ }^{\dagger}$ & NS & Larance [35] & NS & Dunn [15]; Larance [35] \\
\hline Insulin & $\begin{array}{l}\text { Anabolic effects and strength, } \\
\text { burning fat, weight loss }\end{array}$ & $\begin{array}{l}\text { Chandler [12]; Kimergård [30]; } \\
\text { Klötz [31]; Peters [46]; Skårberg } \\
\text { [53,54] }\end{array}$ & $\begin{array}{l}\text { Anabolic effects and strength, } \\
\text { burning fat, weight loss }\end{array}$ & $\begin{array}{l}\text { Chandler [12]; Gårevik [18]; Hope [25]; } \\
\text { Kimergård } \\
\text { [30]; Klötz [31]; Larance [35]; Peters [46]; } \\
\text { Skårberg } \\
\text { [53,54]; Tallon [56] }\end{array}$ \\
\hline $\begin{array}{l}\text { Insulin-like growth factor } \\
1 \text { (IGF-1) }\end{array}$ & $\begin{array}{l}\text { Anabolic effects and strength, } \\
\text { burning fat }\end{array}$ & $\begin{array}{l}\text { Chandler [12]; Klötz [31]; } \\
\text { Lenehan [36]; Peters [46]; } \\
\text { Skårberg [53,54] }\end{array}$ & $\begin{array}{l}\text { Anabolic effects and strength, } \\
\text { burning fat }\end{array}$ & $\begin{array}{l}\text { Chandler [12]; Fudala [17]; Klötz [31]; } \\
\text { Larance [35]; } \\
\text { Lenehan [36]; Peters [46]; Skårberg [53,54] }\end{array}$ \\
\hline Ketamine & NS & Chandler [12] & NS & Chandler [12]; Dunn [15] \\
\hline Laxative & - & - & NS & Goldfield [19] \\
\hline Levodopa & NS & Strauss [55] & Increasing growth hormone & Strauss [55]; Skårberg [54] \\
\hline Lidocaine & NS & Strauss [55] & NS & Strauss [55] \\
\hline Liothyronine & NS & Perry [45] & NS & Perry [45] \\
\hline $\begin{array}{l}\text { Lysergic acid } \\
\text { diethylamide (LSD) }\end{array}$ & NS & Skårberg [54] & NS & Rashid [49]; Skårberg [54] \\
\hline Mechano growth factor & NS & Chandler [12] & NS & Chandler [12] \\
\hline
\end{tabular}


Table 3 Use of non-AAS substances, reason(s)/motive(s) for use, and studies (Continued)

\begin{tabular}{|c|c|c|c|c|}
\hline Melanotan & Boosting training, skin tanning & Chandler [12]; Kimergård [30] & Boosting training, skin tanning & Chandler [12]; Hope [25]; Kimergård [30] \\
\hline Mephedrone & NS & Chandler [12] & NS & Chandler [12] \\
\hline Meth/amphetamine & $\begin{array}{l}\text { Alertness, boosting training, } \\
\text { burning fat, increasing aggression } \\
\text { during exercise, increasing strength, } \\
\text { psychological wellbeing }\end{array}$ & $\begin{array}{l}\text { Chandler [12]; Hegazy [23]; } \\
\text { Kimergård [30]; Larance [35]; } \\
\text { Lundholm [37]; McBride [39]; } \\
\text { Pappa [43]; Peters [46]; } \\
\text { Skårberg [53,54] }\end{array}$ & $\begin{array}{l}\text { Alertness, boosting training, } \\
\text { burning fat, increasing aggression } \\
\text { during exercise, increasing strength, } \\
\text { psychological wellbeing }\end{array}$ & $\begin{array}{l}\text { Angoorani [10]; Chandler [12]; Gårevik } \\
\text { [18]; } \\
\text { Hegazy [23]; Hoff [24]; Hope [25]; } \\
\text { Kimergård } \\
\text { [30]; Larance [35]; Lundholm [37]; } \\
\text { McBride [39]; } \\
\text { Pappa [43]; Peters [46]; Skårberg [53,54]; } \\
\text { Tallon [56] }\end{array}$ \\
\hline Muscle oil (synthol) & - & - & Anabolic effect & Skårberg [54] \\
\hline Muscle relaxing drugs ${ }^{\dagger}$ & Combating side effects & Klötz [31] & Combating side effects & Klötz [31] \\
\hline Myoblast & NS & Klötz [31] & NS & Klötz [31] \\
\hline Nalbuphine/nubain ${ }^{\oplus}$ & NS & $\begin{array}{l}\text { Strauss [55]; Lenehan [36]; } \\
\text { McBride [39] }\end{array}$ & $\begin{array}{l}\text { Treating pain from weightlifting } \\
\text { injuries, } \\
\text { "anti-catabolic", mental high }\end{array}$ & $\begin{array}{l}\text { Gruber [22]; Hope [25]; Kanayama } \\
\text { [27]; Korkia } \\
\text { [33]; Lenehan [36]; McBride [39]; } \\
\text { Strauss [55]; } \\
\text { Wines [58] }\end{array}$ \\
\hline Naproxen & NS & Strauss [55] & NS & Strauss [55] \\
\hline $\begin{array}{l}\text { Non-steroidal anti- } \\
\text { inflammatory drugs } \\
\text { (NSAIDs) }^{\dagger}\end{array}$ & - & - & Inflammation, pain, and fever relief & Skårberg [54] \\
\hline Oxycodone & NS & Strauss [55] & NS & Kanayama [27]; Strauss [55] \\
\hline Peptide hormones ${ }^{\dagger}$ & - & - & NS & Bilard [11] \\
\hline Phenylbutazone & NS & Strauss [55] & NS & Strauss [55] \\
\hline $\begin{array}{l}\text { Phosphodiesterase- } 5 \\
\text { inhibitors }\left(\text { PDE5i) }^{\dagger}\right.\end{array}$ & - & - & NS & Hope [25] \\
\hline Piroxicam & NS & Strauss [55] & NS & Strauss [55] \\
\hline Potassium & NS & Strauss [55] & NS & Strauss [55] \\
\hline $\begin{array}{l}\text { Potency/testicular } \\
\text { increasing drugs }^{\dagger}\end{array}$ & Combating side effects & Klötz [31] & Combating side effects & Klötz [31] \\
\hline 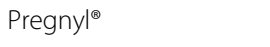 & Improved testosterone production & Peters [46] & Improved testosterone production & Peters [46] \\
\hline Protein powder & $\begin{array}{l}\text { Enhancing effects of training, } \\
\text { increasing protein synthesis }\end{array}$ & Perry [45]; Skårberg [53,54] & $\begin{array}{l}\text { Enhancing effects of training, } \\
\text { increasing protein synthesis }\end{array}$ & Perry [45]; Skårberg [53,54] \\
\hline Proviron $^{\oplus}$ & $\begin{array}{l}\text { Hardiness, improved testosterone } \\
\text { production }\end{array}$ & Peters [46] & $\begin{array}{l}\text { Hardiness, improved testosterone } \\
\text { production }\end{array}$ & Peters [46] \\
\hline Recovery drinks $^{\dagger}$ & - & - & Endurance booster, recovery aid & Filiault [16] \\
\hline Sedatives $^{\dagger}$ & NS & Malone [38] & NS & Malone [38] \\
\hline
\end{tabular}


Table 3 Use of non-AAS substances, reason(s)/motive(s) for use, and studies (Continued)

\begin{tabular}{|c|c|c|c|c|c|}
\hline Sildenafil/viagra $\varpi^{\circledR}$ cialis & Enhanced sexual functioning & Chandler [12]; Kimergård [30] & Enhanced sexual functioning & $\begin{array}{l}\text { Chandler [12]; Gårevik [18]; Hope [25]; } \\
\text { Kimergård [30] }\end{array}$ & 4 \\
\hline Somatotropine & NS & Klötz [31] & NS & Klötz [31] & 1 \\
\hline Spironolactone & Weight loss & Ahlgrim [9] & Weight loss & Ahlgrim [9] & 1 \\
\hline Stimulants $^{\dagger}$ & NS & $\begin{array}{l}\text { Klötz [31]; Kusserow [34]; } \\
\text { Malone [38] }\end{array}$ & Increasing strength, burning fat & $\begin{array}{l}\text { Fudala [17]; Klötz [31]; Kusserow [34]; Malone } \\
\text { [38]; Rashid [49]; Skårberg [54] }\end{array}$ & 6 \\
\hline Suntan pills ${ }^{\dagger}$ & NS & Strauss [55] & NS & Strauss [55] & 1 \\
\hline Tamoxifen & Combating side effects & $\begin{array}{l}\text { Chandler [12]; Kimergård [30]; } \\
\text { Lenehan [36]; McBride [39] }\end{array}$ & Combating side effects & $\begin{array}{l}\text { Chandler [12]; Gruber [22]; Kimergård [30]; } \\
\text { Korkia [33]; Lenehan [36]; McBride [39]; } \\
\text { Tallon [56] }\end{array}$ & 7 \\
\hline Teroxin (T3) & Preventing weight gain & Peters [46] & Preventing weight gain & Peters [46] & 1 \\
\hline $\begin{array}{l}\text { Testosterone releasers/ } \\
\text { boosters }^{\dagger}\end{array}$ & $\begin{array}{l}\text { Combating side effects, increasing } \\
\text { hormone production }\end{array}$ & Skårberg [53] & $\begin{array}{l}\text { Combating side effects, increasing } \\
\text { blood serum levels of testosterone } \\
\text { or hormone production }\end{array}$ & Skårberg [53,54] & 2 \\
\hline Thiazides $^{\dagger}$ & - & - & NS & McKillop [40] & 1 \\
\hline Thiomucase & NS & Wilson-Fearon [57] & Burning fat & Korkia [32,33]; Wilson-Fearon [57] & 3 \\
\hline Thyroxine & $\begin{array}{l}\text { Burning fat, } \\
\text { increasing metabolism }\end{array}$ & $\begin{array}{l}\text { Chandler [12]; Lenehan [36]; } \\
\text { Peters [46]; Skărberg [54]; } \\
\text { Strauss [55] }\end{array}$ & Burning fat, increasing metabolism & $\begin{array}{l}\text { Chandler [12]; Gruber [22]; Hope [25]; Korkia } \\
\text { [32]; Larance [35]; Lenehan [36]; McKillop } \\
\text { [40]; Peters [46]; Skårberg [54]; Strauss [55] }\end{array}$ & 10 \\
\hline Tobacco & NS & $\begin{array}{l}\text { Malone [38]; Perry [48]; Pope } \\
\text { [47] }\end{array}$ & NS & Malone [38]; Perry [48]; Pope [47] & 3 \\
\hline Torsemide & NS & Ahlgrim [9] & NS & Ahlgrim [9] & 1 \\
\hline Triacana & - & - & NS & Korkia [33] & 1 \\
\hline Yohimbine & NS & Perry [45] & NS & Gruber [22]; Perry [45] & 3 \\
\hline $\begin{array}{l}\text { Other IPEDs, licit and } \\
\text { illicit substances }\end{array}$ & NS & $\begin{array}{l}\text { Kanayama [28]; Klötz [31]; } \\
\text { Kusserow [34]; Perry [48]; } \\
\text { Skărberg [52] }\end{array}$ & NS & $\begin{array}{l}\text { Gruber [20,21]; Hoff [24]; Hope [25]; } \\
\text { Kanayama [26,28]; } \\
\text { Klötz [31]; Kusserow [34]; Perry [48]; } \\
\text { Skårberg [52,54]; } \\
\text { Wines [58] }\end{array}$ & \\
\hline
\end{tabular}

IPEDs: Image and performance-enhancing drugs.

NS: Not specified.

${ }^{\dagger}$ Substance class - not specified. 
Table 4 Groups of non-AAS substances used by AAS users

\section{Group}

Analgesics/non-steroidal anti-

inflammatory drugs/opioids

Anti-oestrogens

Cardiovascular drugs

CNS depressants

CNS stimulants

Cosmetic drugs

Dietary/nutritional supplements

Diuretics

Fat burning/weight loss drugs

Muscle/strength-enhancement drugs (non-hormone)

Muscle/strength-enhancement hormones

Recreational substances/drugs

Sexual enhancement drugs

CNS: Centrat newous system.

CNS: Central nervous system.

${ }^{\dagger}$ Substance class - not specified.

- Some are used for direct muscle enhancing properties and others for counteracting shut-down of endogenous testosterone production.

** Skårberg [54].

There may be overlap between classes (e.g. CNS depressants may be used for promoting sleep and for analgesic properties).

Some of the drugs do not have well documented efficacy for their alleged motives for use.

\section{Purpose(s)}

Acetaminophen, aspirin ${ }^{\circledast}$, Ben-Gay ${ }^{\oplus}$, benoxaprofen, buprenorphine, codeine, corticosteroids ${ }^{\dagger}$ heroin, hydrocodone, lidocaine, muscle oil (synthol) and muscle relaxing drugs ${ }^{\dagger}$, nalbuphine/ nubain ${ }^{\oplus}$, naproxen, oxycodone, phenylbutazone, piroxicam

Aminogluthimide, aromatase inhibitors ${ }^{\dagger}$, clomiphene/clomid, proviron ${ }^{\oplus}$, tamoxifen

Beta-2-agonists $^{\dagger}$, beta-blockers ${ }^{\dagger}$, captopril, carvedilol, digoxin, thiazides $^{\dagger}$

Alcohol, benzodiazepines ${ }^{\dagger}$, buprenorphine, cannabis/cannabinoids, diazepam, gamma hydroxybutyrate (GHB), heroin, hydrocodone, ketamine, oxycodone

Amyl nitrate, caffeine, cocaine, ephedrine, epinephrine, mephedrone, meth/amphetamine, yohimbine

Anti-acne drugs ${ }^{\dagger}$, esiclene, melanotan I, suntan pills, thiomucase

Calcium, choline and inositol, chromium picolinate, conjugated linoleic acid, creatine, electrolyte solution, glutamine, hydroxocobal amin, piroxicam, potassium, protein powder

Furosemide, hydrochlorothiazide, spironolactone, torsemide

2,4-dinitrophenol (DNP), anti-oestrogens ${ }^{\dagger}$, beta blockers ${ }^{\dagger}$, bronchodilators $^{\dagger}$, caffeine, chromium picolinate, clenbuterol, cocaine, conjugated linoleic acid, ephedrine, hydrochlorothiazide, insulin ${ }^{* *}$, laxatives ${ }^{\dagger+}$, liothyronine, melanotan II, meth/amphetamine, spironolactone, teroxin (T3), thiomucase, thyroxine, triacana, yohimbine

Amphetamine/meth, anti-catabolics ${ }^{\dagger}$, glutamine, bronchodilators, chromium picolinate, clenbuterol, creatine, ephedrine, herbal products ${ }^{+1}$, hydroxocobal amin (B12), myoblast, muscle oil (synthol), protein powder, recovery drinks

Dehydroepiandrosterone (DHEA), erythropoietin (EPO), genotropine, growth hormone, growth hormone releasing peptide (GHRP), human chorionic gonadotropin (hCG), insulin-like growth factor 1 (IGF-1), insulin, levodopa, mechano growth factor, pregny ${ }^{\oplus}$, prohormones ${ }^{\dagger}$, proviron ${ }^{\oplus}$ somatotropine

Alcohol, buprenorphine, cannabis/cannabinoids, cigarettes/tobacco methamphetamine, blood pressure regulators ${ }^{\dagger}$, caffeine, cocaine, ecstasy, hallucinogens ${ }^{\dagger}$, heroin, hydrocodone, ketamine lysergic acid diethylamide (LSD), sedatives ${ }^{\dagger}$, tetrahydrocannabinol

Anti-oestrogens ${ }^{\dagger}$, human chorionic gonadotropin (hCG), melanotan II, phosphodiesterase-5 inhibitors (PDE5i), sildenafil/cialis ${ }^{\oplus}$, yohimbine
Relieving inflammation, pain, and fever

Improved testosterone production, burning body fat, reducing the effects of AAS on oestrogens, and dealing with gynecomastia

Lowering blood pressure, reducing risk of infarction, and burning body fat

Improving sleep, relaxation, and dealing with side effects

of AAS use such as gynecomastia

Alertness, boosting training, burning body fat, increased aggression and strength, and psychological wellbeing

Curing acne, skin tanning, and enhancing physical appearance

For essential nutrients to supplement the diet and combat the risk of illness

Increasing strength, masking AAS and other doping drugs, burning body fat, and reducing levels of body fluid

Suppression of appetite, increased metabolism, and reduced absorption of body fat

Enhancing the size and structure of muscles as well as boosting strength

Enhancing the size and structure of muscles as well as boosting strength

Enhancing sleep, relaxation, and psychological wellbeing

Dealing with testicular atrophy, improved sexual desire or arousal and boosting erectile functioning 


\section{Implications for policy and practice}

The present study has highlighted various licit and illicit substances used by AAS users. Evidence abounds that some of the substances identified in our study, especially dietary and nutritional supplements, may be contaminated with AAS and other pharmacological elements thus, potentially, playing a role in the decision to initiate AAS use [2,64-67]. Preventive efforts should therefore highlight the potential role licit and illicit substance use, especially dietary and nutritional supplement use, may play in the initiation of AAS use as well as the role AAS use may potentially play in the use of other substances, together with the potential negative consequences individuals who engage in such behavior may encounter.

AAS-associated polypharmacy is dangerous for several reasons. First, it has been associated with violent and criminal behavior as well as various forms of pathology and mortality [68-70]. Second, chemical interactions from AAS-related polypharmacy may have adverse psychophysical effects on individuals engaged in such behavior. Thus, the main and combined effects of the use of these substances must attract the attention of clinicians, policymakers and public health officials. Indeed, physicians may inadvertently administer medication to AAS-using polydrug users thereby triggering unintended adverse chemical interactions that may be harmful to AAS-using patients. Accordingly, gathering correct and comprehensive substance use histories of AAS users is important in the effective pharmacological and psychological treatment of AAS users [67,71] as such information may guide clinicians in the diagnosis and prescription of 'safe' drugs during treatment.

Additionally, most AAS users obtain the substances identified in the present study from the illicit market $[1,2]$. Because many of these substances are controlled or illegal [2], they may be produced in unsterile 'underground laboratories' leading to inadvertent and sometimes deliberate incorrect dosing, substitution of ingredients and contamination with additional pharmaceuticals, toxic chemicals and pathogens. Furthermore, some users resort to unsterile injection equipment for the administration of these products, resulting in injecting site injuries as well as bacterial and fungal infection [72] and the potential transmission of blood borne viruses such as hepatitis B/C and HIV [25]. Stakeholders must take our findings into consideration in the development of preventive and therapeutic interventions for AAS users. There is also the need for the strengthening of harm reduction interventions to combat the harmful consequences of AAS-related polydrug use.

\section{Implications for research}

There is the need for further investigations to elucidate better the pathway to AAS-associated polysubstance use. Further studies are also necessary to examine the main and complementary enervating consequences of the use of different dosages of these varied substances, plus their addictive potential and trajectories. Moreover, there is a dearth of knowledge regarding the spread of these substances due to the fact that most of these substances are relatively new. So far most focus has been directed toward AAS in particular. Thus, the use of ancillary and associated substances has mainly escaped the attention of clinicians, public health officials, policymakers, and researchers [2]. There is therefore the need for studies examining the emergence of these substances in the pharmacopoeia of substance users as well as their diffusion into other substance-using populations.

There is the need for the collection and analysis or testing of these substances, to ascertain their content and potential contaminants. Additionally, apart from the Iranian study [10], all studies were conducted in Western countries. Nonmedical AAS use is a global public health problem [4] and researchers are encouraged to extend their investigations to non-Western nations. Finally, investigations of AAS-associated polypharmacy must be a continuous process requiring updates as evidence accumulates.

\section{Strengths and limitations}

As far as we are aware, the present study is the pioneering international systematic review and synthesis of qualitative studies on AAS use and polypharmacy. The inclusion of both peer-reviewed and grey literature, as well as literature published before 1995 and after 2009, also distinguishes this review from a previous review [3]. The present study also has some limitations that ought to be taken into consideration when interpreting our findings. First, due to the nature of the present study, it was not statistically possible to establish 'gateway' or causal associations between AAS use and use of the other substances. In addition, we were unable to establish the prevalence of the use of these substances by AAS users. Third, some of the studies included in the present study did not specifically investigate AAS users' intake of other licit and illicit substances. Although these studies present very useful data in respect of the present study, it is plausible that these studies do not present a comprehensive picture of the variety of substances ingested by AAS users. Similarly, the case reports included in the present study may have been published because they are 'extraordinary' and may therefore not be representative of the 'typical' AAS user. With the relative paucity of literature in this field [73], the inclusion of these studies is in our view still defendable. Finally, there is the possibility that our exclusion of non-English language literature may have biased our results. It should be noted however that this very common practice in terms of reviews and meta-analyses might not necessarily affect findings [62]. 


\section{Conclusions}

Our findings corroborate previous suggestions of associations between AAS use and the use of a wide range of other licit and illicit substances. AAS-related polypharmacy has potential serious harmful effects for persons who engage in such behavior, which should be of serious public health concern. Clinicians, policymakers, researchers, and public health workers dealing with AAS users must be educated about these issues. Importantly, efforts must be intensified to combat the debilitating effects of AASconcomitant polypharmacy. Furthermore, there needs to be ongoing research to investigate trends in AAS use and polypharmacy.

\section{Competing interests}

The authors declare that they have no competing interests.

\section{Authors' contributions}

DS led the conception and design of the study, the literature search, analysis, and writing of the manuscript. JM, AB, M-SE, CSA, and SP contributed to the analysis and writing of the manuscript. All authors read and approved the final manuscript.

\section{Authors' information}

DS is PhD research fellow at the Department of Psychosocial Science, University of Bergen, Norway. He conducts research on image and performance enhancing drugs and methods with special focus on anabolic-androgenic steroids. JM is Acting Director at the Centre for Public Health, Liverpool John Moores University, United Kingdom. He has an academic interest in all licit and illicit substance use and has investigated the public health implications of anabolic steroid use for the last 20 years. AB is a postdoctoral research fellow at the Department of Physical Medicine and Rehabilitation, Unit of Neuropsychology, Oslo University Hospital, Norway. She conducts research on long-term anabolic steroid use and effects on brain morphometry, cognitive function, and emotional processing. M-SE is a medical doctor at the Departments of Surgery and Paediatrics, La General Hospital in Accra, Ghana. CSA is a postdoctoral research fellow at the Department of Psychosocial Science, University of Bergen, Norway, and a clinical psychologist specialist at the Bergen Clinics Foundation, Norway. She conducts research in the area of work, industrial and organizational psychology, as well as chemical and non-chemical addictions. SP is a professor of psychology at the Department of Psychosocial Science, University of Bergen, Norway, and a senior researcher at the Norwegian Competence Centre for Sleep Disorders. He conducts research on sleep and sleep disorders as well as chemical and non-chemical addictions.

\section{Acknowledgements}

We are grateful to Philomena Antwi for reviewing studies included in the metasynthesis.

\section{Author details \\ ${ }^{1}$ Department of Psychosocial Science, University of Bergen, Christiesgate 12, 5015 Bergen, Norway. ${ }^{2}$ Centre for Public Health, Liverpool John Moores University, 15-21 Webster Street, Liverpool L3 2ET, UK. ${ }^{3}$ Department of Physical Medicine and Rehabilitation, Unit of Neuropsychology, Oslo University Hospital, Kirkeveien 166, Ullevål, Norway. ${ }^{4}$ Departments of Surgery and Paediatrics, La General Hospital, PMB, Accra, Ghana. ${ }^{5}$ The Competence} Centre, Bergen Clinics Foundation, Vestre Torggate 11, 5015 Bergen, Norway.

\section{Received: 19 December 2014 Accepted: 24 February 2015}

Published online: 15 March 2015

\section{References}

1. Sagoe D, Andreassen CS, Pallesen S. The aetiology and trajectory of anabolic-androgenic steroid use initiation: a systematic review and synthesis of qualitative research. Subst Abuse Treat Prev Policy. 2014;9:27.
2. Evans-Brown M, McVeigh J, Perkins C, Bellis MA. Human enhancement drugs: the emerging challenges to public health. Liverpool: North West Public Health Observatory; 2012.

3. Dodge T, Hoagland MF. The use of anabolic androgenic steroids and polypharmacy: a review of the literature. Drug Alcohol Depend. 2011;114:100-9.

4. Sagoe D, Molde H, Andreassen CS, Torsheim T, Pallesen S. The global epidemiology of anabolic-androgenic steroid use: a meta-analysis and meta-regression analysis. Ann Epidemiol. 2014;24:383-98.

5. Shaw R, Booth A, Sutton A, Miller T, Smith JA, Young B, et al. Finding qualitative research: an evaluation of search strategies. BMC Med Res Methodol. 2004:4:5.

6. Moher D, Liberati A, Tetzlaff J, Altman DG, The PRISMA Group. Preferred reporting items for systematic reviews and meta-analyses: the PRISMA statement. PLOS Med. 2009;6:e1000097.

7. Smith JA, Harré R, Van Langenhove L. Idiography and the case study. In: Smith JA, Harre R, Van Langenhove L, editors. Rethinking psychology. London: Sage; 1995. p. 57-69.

8. Corp IBM. IBM SPSS statistics for windows, version 20.0. Armonk, NY: IBM Corp; 2011.

9. Ahlgrim C, Guglin M. Anabolics and cardiomyopathy in a bodybuilder: case report and literature review. J Card Fail. 2009;15:496-500.

10. Angoorani H, Narenjiha H, Tayyebi B, Ghassabian A, Ahmadi G, Assari S. Amphetamine use and its associated factors in body builders: a study from Tehran, Iran. Arch Med Sci. 2012;8:362-7.

11. Bilard J, Ninot G, Hauw D. Motives for illicit use of doping drugs among athletes calling a national antidoping phone-help service: an exploratory study. Subst Use Misuse. 2011;46:359-67.

12. Chandler M, McVeigh J. Steroids and image enhancing drugs 2013 survey results. Liverpool: LUMU Centre for Public Health; 2014.

13. Cornford CS, Kean J, Nash A. Anabolic-androgenic steroids and heroin use: a qualitative study exploring the connection. Int J Drug Policy. 2014;25:928-30.

14. Davies R, Smith D, Collier K. Muscle dysmorphia among current and former steroid users. J Clin Sport Psychol. 2011;5:77-94.

15. Dunn M. The non-medical use of steroids in Australia: results from a general population survey. Aust N Z J Public Health. 2010;34:531-2.

16. Filiault SM, Drummond MJ. Muscular, but not 'roided out': gay male athletes and performance-enhancing substances. Int J Mens Health. 2010;9:62-81.

17. Fudala PJ, Weinrieb RM, Calarco JS, Kampman KM, Boardman C. An evaluation of anabolic-androgenic steroid abusers over a period of 1 year: seven case studies. Ann Clin Psychiatry. 2003;15:121-30.

18. Gårevik N, Rane A. Dual use of anabolic-androgenic steroids and narcotics in Sweden. Drug Alcohol Depend. 2010;109:144-6.

19. Goldfield GS. Body image, disordered eating and anabolic steroid use in female bodybuilders. Eat Disord. 2009;17:200-10.

20. Gruber AJ, Pope Jr HG. Ephedrine abuse among 36 female weightlifters. Am J Addict. 1998:7:256-61.

21. Gruber AJ, Pope Jr HG. Compulsive weight lifting and anabolic drug abuse among women rape victims. Compr Psychiatry. 1999:40:273-7.

22. Gruber AJ, Pope Jr HG. Psychiatric and medical effects of anabolic-androgenic steroid use in women. Psychother Psychosom. 2000;69:19-26.

23. Hegazy B, Sanda C. A 28-year-old man with depression, PTSD, and anabolic-androgenic steroid and amphetamine use. Psychiatr Ann. 2013:43:408-11

24. Hoff D. Doping, risk and abuse: an interview study of elite athletes with a history of steroid use. Perform Enhanc Health. 2012;1:61-5.

25. Hope VD, McVeigh J, Marongiu A, Evans-Brown M, Smith J, Kimergård A, et al. Prevalence of, and risk factors for, HIV, hepatitis B and C infections among men who inject image and performance enhancing drugs: a crosssectional study. BMJ Open. 2013;3:e003207.

26. Kanayama G, Pope Jr HG, Cohane G, Hudson Jl. Risk factors for anabolic-androgenic steroid use among weightlifters: a case-control study. Drug Alcohol Depend. 2003;71:77-86.

27. Kanayama G, Cohane GH, Weiss RD, Pope Jr HG. Past anabolic-androgenic steroid use among men admitted for substance abuse treatment: an underrecognized problem? J Clin Psychiatry. 2003;64:156-60.

28. Kanayama G, Hudson Jl, Pope Jr HG. Features of men with anabolic-androgenic steroid dependence: a comparison with nondependent AAS users and with AAS nonusers. Drug Alcohol Depend. 2009;102:130-7. 
29. Katz DL, Pope Jr HG. Anabolic-androgenic steroid-induced mental status changes. NIDA Res Monogr. 1990;102:215-23.

30. Kimergård A. A qualitative study of anabolic steroid use amongst gym users in the United Kingdom: motives, beliefs and experiences. J Subst Use 2014 doi: 10.3109/14659891.2014.911977. [Epub ahead of print].

31. Klötz F, Petersson A, Hoffman O, Thiblin I. The significance of anabolic androgenic steroids in a Swedish prison population. Compr Psychiatry. 2010;51:312-8.

32. Korkia P, Stimson G. Anabolic steroid use in Great Britain: an exploratory investigation. Final report to the Department of Health for England, Scotland and Wales. London: The Centre for Research on Drugs and Health Behaviour; 1993.

33. Korkia P, Lenehan P, McVeigh J. Non-medical use of androgens among women. J Perform Enhanc Drugs. 1996;1:71-6.

34. Kusserow RP. Adolescents and steroids: a user perspective. Washington, DC: Office of Inspector General; 1990.

35. Larance B, Degenhardt L, Copeland J, Dillon P. Injecting risk behaviour and related harm among men who use performance-and image-enhancing drugs. Drug Alcohol Rev. 2008;27:679-86.

36. Lenehan P, Bellis M, McVeigh J. A study of anabolic steroid use in the North West of England. J Perform Enhanc Drugs. 1996;1:57-70.

37. Lundholm L, Käll K, Wallin S, Thiblin I. Use of anabolic androgenic steroids in substance abusers arrested for crime. Drug Alcohol Depend. 2010;111:222-6.

38. Malone Jr DA, Dimeff RJ, Lombardo JA, Sample RB. Psychiatric effects and psychoactive drug use in anabolic-androgenic steroid users. Clin J Sport Med. 1995;5:25-31.

39. McBride AJ, Williamson K, Petersen T. Three cases of nalbuphine hydrochloride dependence associated with anabolic steroid use. $\mathrm{Br} J$ Sports Med. 1996;30:69-70.

40. McKillop G. Drug abuse in bodybuilders in the west of Scotland. Scott Med J. 1987;32:39-41.

41. Moss HB, Panzak GL, Tarter RE. Personality, mood, and psychiatric symptoms among anabolic steroid users. Am J Addict. 1992;1:315-24.

42. Moss HB, Panzak GL, Tarter RE. Sexual functioning of male anabolic steroid abusers. Arch Sex Behav. 1993;22:1-12.

43. Pappa E, Kennedy E. "It was my thought ... he made it a reality': normalization and responsibility in athletes' accounts of performance enhancing drug use. Int Rev Sociol Sport. 2012;48:277-94.

44. Perry PJ, Andersen KH, Yates WR. Illicit anabolic steroid use in athletes. A case series analysis. Am J Sports Med. 1990;18:422-8.

45. Perry PJ, Kutscher EC, Lund BC, Yates WR, Holman TL, Demers L. Measures of aggression and mood changes in male weightlifters with and without androgenic anabolic steroid use. J Forensic Sci. 2003;48:646-51.

46. Peters R, Copeland J, Dillon P, Beel A. Patterns and correlates of anabolic-androgenic steroid use. Technical Report No 48. Sydney: National Drug and Alcohol Research Centre; 1997.

47. Pope Jr HG, Katz DL. Affective and psychotic symptoms associated with anabolic steroid use. Am J Psychiatry. 1988;1:487-90.

48. Pope Jr HG, Katz DL. Psychiatric and medical effects of anabolic-androgenic steroid use: a controlled study of 160 athletes. Arch Gen Psychiatry. 1994;51:375-82.

49. Rashid W. Testosterone abuse and affective disorders. J Subst Abuse Treat. 2000:18:179-84.

50. Schäfer CN, Guldager H, Jørgensen HL. Multi-organ dysfunction in bodybuilding possibly caused by prolonged hypercalcemia due to multi-substance abuse: case report and review of literature. Int J Sports Med. 2011;32:60-5.

51. Silvester LJ. Self-perceptions of the acute and long-range effects of anabolic-androgenic steroids. J Strength Cond Res. 1995;9:95-8.

52. Skårberg K, Engstrom I. Troubled social background of male anabolic-androgenic steroid abusers in treatment. Subst Abuse Treat Prev Policy. 2007;2:20.

53. Skårberg K, Nyberg F, Engström I. The development of multiple drug use among anabolic-androgenic steroid users: six subjective case reports. Subst Abuse Treat Prev Policy. 2008;3:24.

54. Skårberg K, Nyberg F, Engström I. Multisubstance use as a feature of addiction to anabolic-androgenic steroids. Eur Addict Res. 2009;15:99-106.

55. Strauss RH, Liggett MT, Lanese RR. Anabolic steroid use and perceived effects in ten weight-trained women athletes. JAMA. 1985;253:2871-3.

56. Tallon V. An exploratory investigation of anabolic-androgenic steroid use in Lanarkshire. Paisley: University of Paisley; 2007. Retrieved June 2014 from http://www.ibrarian.net/navon/page.jsp?paperid=10804537\&search Term=east+kilbride.
57. Wilson-Fearon C, Parrott AC. Multiple drug use and dietary restraint in a Mr. Universe competitor: psychobiological effects. Percept Mot Skills. 1999;88:579-80.

58. Wines JD, Gruber AJ, Pope Jr HG, Lukas SE. Nalbuphine hydrochloride dependence in anabolic steroid users. Am J Addict. 1999;8:161-4.

59. Norwegian Institute of Public Health: Alcohol and other drugs in Norway - Public health report 2014 [Alkohol og andre rusmidler i Norge - Folkehelserapporten 2014]. Oslo; 2014. Retrieved from http://www.fhi.no/artikler/?id=110521

60. Clauson KA, Polen HH, Boulos MNK, Dzenowagis JH. Scope, completeness, and accuracy of drug information in Wikipedia. Ann Pharmacother. 2008:42:1814-21

61. Viera AJ, Garrett JM. Understanding interobserver agreement: the kappa statistic. Fam Med. 2005;37:360-3.

62. Moher D, Pham B, Klassen TP, Schulz KF, Berlin JA, Jadad AR, et al. What contributions do languages other than English make on the results of meta-analyses? J Clin Epidemiol. 2000;53:964-72.

63. Evans-Brown M, Kimergård A, McVeigh J. Elephant in the room? The methodological implications for public health research of performance-enhancing drugs derived from the illicit market. Drug Test Anal. 2009;1:323-6.

64. Hildebrandt T, Harty S, Langenbucher JW. Fitness supplements as a gateway substance for anabolic-androgenic steroid use. Psychol Addict Behav. 2012;26:955-62.

65. Geyer H, Parr MK, Koehler K, Mareck U, Schanzer W, Thevis M. Nutritional supplements cross-contaminated and faked with doping substances. J Mass spectrom. 2008:43:892-902

66. Hoffman JR, Faigenbaum AD, Ratamess NA, Ross R, Kang J, Tenenbaum G. Nutritional supplementation and anabolic steroid use in adolescents. Med Sci Sports Exerc. 2008;40:15-24.

67. Kimergård A, Breindahl T, Hindersson P, McVeigh J. The composition of anabolic steroids from the illicit market is largely unknown: implications for clinical case reports. QJM 2014, doi:10.1093/qjmed/hcu101.

68. Chahla E, Hammami MB, Befeler AS. Hepatotoxicity associated with anabolic androgenic steroids present in over-the-counter supplements: a case series. Int J Appl Sci Technol. 2014:4:179-83.

69. Darke S, Torok M, Duflou J. Sudden or unnatural deaths involving anabolic-androgenic steroids. J Forensic Sci. 2014;59:1025-8.

70. Pagonis TA, Angelopoulos NV, Koukoulis GN, Hadjichristodoulou CS. Psychiatric side effects induced by supraphysiological doses of combinations of anabolic steroids correlate to the severity of abuse. Eur Psychiatry. 2006;21:551-62.

71. Dawson RT. Drugs in sport-The role of the physician. J Endocrinol. 2001;170:55-61.

72. Hope VD, McVeigh J, Marongiu A, Evans-Brown M, Smith J, Kimergård A, et al. Injection site infections and injuries among men who inject image and performance enhancing drugs: prevalence, risks factors, and healthcare seeking. Epidemiol Infect 2015;143:132-40.

73. Degenhardt L, Hall W. The extent of illicit drug use, dependence, and their contribution to global burden of disease. Lancet. 2012;379:55-70.

\section{Submit your next manuscript to BioMed Central and take full advantage of:}

- Convenient online submission

- Thorough peer review

- No space constraints or color figure charges

- Immediate publication on acceptance

- Inclusion in PubMed, CAS, Scopus and Google Scholar

- Research which is freely available for redistribution 